\title{
Why the Prominent Improvement in Prehospital Medical Response in Iran Couldn't Decrease the Number of Death Related Road Traffic Injuries
}

\section{Hamid Reza Khankeh ${ }^{1,2 *}$, Davoud Khorasani-Zavareh ${ }^{3}$ and Gholamreza Masoumi ${ }^{4}$}

${ }^{1}$ Department of Nursing, University of Social Welfare and Rehabilitation Sciences, Tehran, Iran.

${ }^{2}$ Department of Clinical Science and Education, Karolinska Institute, Stockholm, Sweden

${ }^{3}$ Urmia University of Medical Sciences, Oroumiyeh, Iran

${ }^{4}$ Department of Emergency Medicine, Tehran University of Medical Sciences, Tehran, Iran.

Road Traffic Injuries (RTIs) are still a major public health problem especially in low- and middle-income countries [1]. It caused more than 1.27 million deaths and almost 50 million injuries every year worldwide. For reduction of their consequences it required concerned efforts both for crash prevention and improvement in post crash management $[2,3]$. Iran ranks as one of the highest mortality rates of RTIs in middle-income countries. Focusing on post crash event, studies in Iran have shown that about $60 \%$ of the deaths use to be occurred at the crash scene or during transporting to hospital [2], but the pattern was changed in recent year and about $40 \%$ of the deaths occur in the pre-hospital phase. Changing in this pattern may because of pre-hospital medical response capabilities in recent years and a dramatically improvement in this area including staff training and their skill as well as ambulance number and ambulance dispatch sites and their equipments [4]. Despite these efforts, mortality and morbidity of RTIs has not shown a noticeable decrease [2].

Focusing on factors related to the pre-hospital care of RTIs, recent studies in Iran revealed that two groups of factors can inhibit an efficient pre-hospital trauma care in the context: factors inside and outside the Emergency Medical Services (EML) [2]. Administration and organization, staff qualifications and competences, availability and distribution of resources and communication and transportation are factors inside the EMS and other organizations involvement, layman and the shortcoming infrastructure are factors outside the EMS that influence the process. Interaction and common understanding was identified as the basic social problem [2]. Most facilities were emphasized and were planned by policy makers [1].

Now the question is why in spite of above mentioned improvement in post crash management as well as some policy interventions including compulsory in wearing seat belt, increasing penalties and tougher enforcement for dangerous driving behaviors, and prominent improvement in EMS, crash-related mortality and morbidity have not shown a noticeable decrease $[1,2,4]$.

Considering all aspect of Haddon's Matrix, we think this controversy might be because of a lack of system approach on RTIs prevention and more focus on human factors [1]. The number of injuries and deaths however depends largely on changes to the transportation infrastructure [4]. We believe that environmental factor such as road design, improvement in standards of vehicle safety, focusing on crashworthiness as well as research areas are crucial [1,2]. Changing in stakeholders' attitude with regard to road user safety development should be emphasised [1]. Safety education programmes should focus on defining the behavior. Finally improving the interaction within the current pre-hospital trauma care system and building a common understanding of the role of the Emergency Medical Services is s key factor in developing an effective pre-hospital trauma care system [2].

\section{References}

1. Khorasani-Zavareh D, Mohammadi R, Khankeh HR, Laflamme L, Bikmoradi $A$ et al. (2009) The requirements and challenges in preventing of road traffic injury in Iran. A qualitative study. BMC Public Health 9: 486.

2. Haghparast-Bidgoli $H$, Hasselberg $M$, Khankeh $H$, Khorasani-Zavareh $D$ Johansson E (2010) Barriers and facilitators to provide effective pre-hospital trauma care for road traffic injury victims in Iran: a grounded theory approach. BMC Emerg Med 10: 20

3. http://www.who.int/healthinfo/global_burden_disease/GBD report_2004update_full.pdf

4. Khorasani-Zavareh D, Khankeh HR, Mohammadi R, Laflamme L, Bikmorad A, et al. (2009) Post-crash management of road traffic injury victims in Iran. Stakeholders' views on current barriers and potential facilitators. BMC Emerg Med 9: 8.

*Corresponding author: Hamid Reza Khankeh, Associate Professor, Department of Nursing, University of Social Welfare and Rehabilitation Sciences, Tehran, Iran, Tel. +46 7040852 05; Fax: + 468616 2933; E-mail: hamid.khankeh@ki.se

Received April 18, 2012; Accepted April 20, 2012; Published April 21, 2012

Citation: Khankeh HR, Khorasani-Zavareh D, Masoumi G (2012) Why the Prominent Improvement in Prehospital Medical Response in Iran Couldn' Decrease the Number of Death Related Road Traffic Injuries. J Trauma Treat 1:e103. doi:10.4172/2167-1222.1000e103

Copyright: (C) 2012 Khankeh HR, et al. This is an open-access article distributed under the terms of the Creative Commons Attribution License, which permits unrestricted use distribution, and reproduction in any medium, provided the original author and source are credited. 